\title{
Cancer Bone Metastasis, Experimental Study
}

\section{Da-Yong Lu* and Bin Xu}

School of Life Sciences, Shanghai University, Shanghai, China

*Corresponding Author: Da-Yong Lu, School of Life Sciences, Shanghai University, Shanghai, China.
Received: October 26, 2021

Published: November 01, 2021

(C) All rights are reserved by Da-Yong Lu and

Bin Xu.

\begin{abstract}
Cancer bone metastasis was a common clinical evidence for cancers, especially to categories of breast, prostate and lung cancer (general incidence $>30 \%$ ). To avoid further devastating event and human mortality, drug treatment was supposed to intervene. To the promotion and paradigms of clinical drug treatment, experimental study is indispensable. This editorial discusses bone cancer metastasis treatment in experimental study.
\end{abstract}

Keywords: Bone Cancer; Neoplasm Metastasis; Drug Treatment; Tumor Models; Experiment Study

\section{Introduction}

Cancer is the secondary leading cause of human mortality worldwide [1-4]. Cancer bone metastasis is one of frequent cancer metastasis events and mortality in the clinic, especially to categories of breast, prostate and lung cancer (general incidence $>30 \%$ ) [5-9]. To avoid devastating pathogenic consequences and human mortality, the experimental drug treatment study and investigations should be focused. This editorial discusses the landscape of drug development in experimental scenarios.

\section{Experimental cancer models}

There are a lot of different anticancer drugs in the clinic. How to evaluate drug responses is determined by experimental modelsin vitro and in vivo. Several tumor models for bone diseases are included [10-18]:

\section{- In vitro tumor models}

- Conventional tumor cell lines cultured in two dimension or molecular biomarkers involving bone cancer progresses [11-13].

- $\quad$ Miniature drug response models in 3-dimension or microfluidics [12-14].
- Drug targets and mechanisms against tumor stemcells (organoids) [10-14].

- $\quad$ Tumor spectral analysis [15-17].

\section{- In vivo tumor models}

- Spontaneous bone metastases models (subcutaneous, rare incidence in current technology) [18].

- Artificial bone metastases models (iv, cardiovascular, and spinal tumor injection) [18].

- New mechanisms, targets and technology should be progressed step by step $[19,20]$.

- Pharmacological investigation for anti-metastatic drugs [21-24].

- Diagnostic specificity for drug response prediction and evaluation (fluorescence or blood biomarker) [9].

Drug response association between experiments and clinics

- Herbal medicine may be evaluated in physiological conditions and integrity in animals [25-27]. 
- $\quad$ Personalized medicine is an useful drug selection paradigm that may optimize drug treatment against cancer growth and metastasis [28-33]. These knowledge translation could be used in experimental and clinical study.

- Drug combination commonly promote clinical outcomes yet mechanisms should be understood [34,35].

- Pharmaceutical innovation should be evaluated in animal modality, such as nanomedicine and other drug delivery systems [36].

\section{Discussion}

There are many rooms to improve in cancer bone metastatic study (diagnostic, pathology and therapeutics). More mimic experimental tumor models to clinical situations should be built in present experimental study. By update experimental models, clinical success may be reached.

\section{Future Trends}

Experimental study of cancer bone metastasis plays key role for clinical therapeutics and patient's survivals. New pathways should be explored to enrich targets and mechanisms in experimental settings. Knowledge should be accumulation in animals instead of human beings for its medical significance could be easily noticed. Many new discoveries could be expected from these experimental investigations and preclinical evaluation.

\section{Bibliography}

1. Lambert AW., et al. "Emerging biological principles of metastasis". Cell 168 (2017): 670-691.

2. Ahmad AS., et al. "Trends in the lifetime risk of developing cancer in Great Britain; Comparison of risk for those born from 1930-1960". British Journal of Cancer 112 (2015): 943-947.

3. Mehlen P and Puisieux A. "Metastasis; a question of life or death". Nature Reviews Cancer 6 (2006): 449-458.

4. Weidenfeld K and Barkan D. "EMT and stemness in tumor dormacy and outgrowth: Are they intertwined processes?" Frontiers in Oncology 8 (2018): 381.

5. Lu DY and Xu B. "Bone metastasis treatment, major frontiers". Acta Scientific Orthopaedics 4.7 (2021): 1-2.

6. Hakim BAA. "Benign bone tumors, an overview". Acta Scientific Orthopaedics 4.10 (2021): 1-2.
7. Lu DY and Xu B. "Bone cancer and metastatic trials, drug treatment”. Acta Scientific Orthopaedics 4.9 (2021): 31-33.

8. Lu DY., et al. "Cancer Metastasis treatments". Current Drug Therapy 8.1 (2013): 24-29.

9. Bhadresha KP., et al. "A predictive biomarker panel for bone metastasis, liquid biopsy approach". Journal of Bone Oncology 29 (2021): 100371.

10. Lu DY., et al. "Anticancer drug sensitivity testing, a historical review and future perspectives". Current Drug Therapy 10.1 (2015): 44-55.

11. Volm M and Efferth T. "Prediction of cancer drug resistance and implications for personalized medicine". Frontiers in Oncology (2015): 282.

12. Lu DY and Lu TR. "Drug sensitivity testing, a unique drug selection strategy". Advances in Biomarker Sciences and Technology 2 (2020): 59-66.

13. Montero J., et al. "Drug-induced death signaling strategy rapidly predicts cancer response to chemotherapy". Cell 160 (2015): 977-989.

14. Popova AA and Levkin PA. "Precision medicine in oncology: In vitro drug sensitivity and resistance test (DSRT) for selection of personalized anticancer therapy". Advanced Therapeutics (2020): 1900100.

15. Zhang Y., et al. "Anticancer drug sensitivity assay with quantitative heterogeneity testing using single-cell Raman Spectroscope". Molecules 23.11 (2018): 2903.

16. Wang JK., et al. "In vitro anticancer drug sensitivity sensing through single-cell Raman Spectroscopy". Biosensors 11.8 (2021): 286.

17. Hammoud MK., et al. "Raman micro-spectroscopy monitors acquired resistance to targeted cancer therapy at the cellular level". Scientific Reports 8 (2018): 15278.

18. Jelgersma $\mathrm{C}$ and Vajkoczy P. "How to target spinal metastasis in experimental research: An overview of currently used experimental mouse model and future prospects". International Journal of Molecular Sciences 22.11 (2021): 5420.

19. Pantano F., et al. "Integrin alpha 5 in human breast cancer is a mediator of bone metastasis and a therapeutic target for the treatment of osteolytic lesions". Oncogene 40 (2021): 12841299. 
20. Lu DY and Che JY. "Bone disease treatments, importance of technical supports”. Acta Scientific Orthopaedics 4.4 (2021): 55-57.

21. Lu DY., et al. "Anti-metastatic drug development, work out towards new direction". Medicinal Chemistry 8.7 (2018): 512.

22. Lu DY and Lu TR. "Antimetastatic activities and mechanisms of bisdioxopiperazine compounds". Anti-Cancer Agent Medicinal Chemistry 10.7 (2010): 564-570.

23. Zhu H., et al. "DJ-1 mediates the resistance of cancer cells to dihydroarteminisinin through cancer cells through reactive oxygen species removal". Free Radical Biology and Medicine 71 (2014): 121-132.

24. Lu DY., et al. "Development of antimetastatic drugs by targeting tumor sialic acids". Scientia Pharmaceutica 80.3 (2012): 497-508.

25. Agarwal N., et al. "Natural herbs as anticancer drugs". International Journal of Pharm Tech Research 40.3 (2012): 1142-1153.

26. Lu DY., et al. "Natural drug cancer treatment strategies from herbal medicine to chemical or biological drug". Studies in Natural Products Chemistry 66 (2020): 91-115.

27. Lu DY and Lu TR. "Herbal medicine in new era". Hospice Palliative Medicine International Journal 3.4 (2019): 125-130.

28. Lu DY., et al. "Individualized cancer chemotherapy integrating drug sensitivity tests, pathological profile analysis and computational coordination-an effective strategy to improve clinical treatment". Medical Hypotheses 66.1 (2006): 45-51.

29. Lu DY. "Personalized cancer chemotherapy, an effective way for enhancing outcomes in clinics (2014).

30. Lu DY., et al. "Cancer bioinformatics for update anticancer drug developments and personalized therapeutics". Reviews on Recent Clinical Trials 12.2 (2017): 101-110.

31. Lu DY., et al. "Pharmacogenetics of cancer therapy: breakthroughs from beyond?" Future Science OA 1.4 (2015): 80.

32. Hyman DH., et al. "Implementing genome-driven oncology". Cell 168 (2017): 584-599.

33. Lu DY., et al. "Individualized cancer therapy, future approaches". Current Pharmacogenomics and Personalized Medicine 16.2 (2018): 156-163.
34. Lu DY., et al. "Anticancer drug combination, how far we can go through?" Anti-Cancer Agents in Medicinal Chemistry 17.1 (2017): 21-28.

35. Lu DY., et al. "Drug combination in clinical cancer treatment". Reviews on Recent Clinical Trials 12.3 (2017): 202-211.

36. Barani M., et al. "Recent advances in nanotechnology-based diagnosis and treatments of human osteosarcoma". Biosensors 11 (2021): 55.

\section{Volume 4 Issue 12 December 2021 \\ (C)All rights are reserved by Da-Yong Lu and Bin Xu.}

